\title{
New insights into molecular mechanisms of sunitinib-associated side effects
}

\author{
Guadalupe Aparicio-Gallego ${ }^{1}$, Moisés Blanco ${ }^{1}$, Angélica Figueroa ${ }^{1}$, Rosario García- \\ Campelo ${ }^{1}$, Manuel Valladares-Ayerbes ${ }^{1}$, Enrique Grande-Pulido ${ }^{2}$, and Luis Antón- \\ Aparicio $^{1}$ \\ ${ }^{1}$ Clinical Oncology Department and Biomedical Research Institute (INIBIC), A Coruña University Hospital, A \\ Coruña; \\ ${ }^{2}$ Clinical Oncology Department, Ramón y Cajal University Hospital, Madrid, Spain
}

\begin{abstract}
The introduction of targeted therapy represents a major advance in the treatment of tumor progression. Targeted agents are a novel therapeutic approach developed to disrupt different cellular signaling pathways. The tyrosine kinase inhibitor sunitinib specifically blocks multiple tyrosine kinase receptors that are involved in the progression of many tumors. Sunitinib is the current standard of care in first-line treatment of advanced renal cell carcinoma, and it is approved in imatinib-intolerant and imatinib-refractory gastrointestinal stromal tumors. However, it is increasingly evident that sunitinib may display collateral effects on other proteins beyond its main target receptors, eliciting undesirable and unexpected adverse events. A better understanding of the molecular mechanisms underlying these undesirable sunitinib-associated side effects will help physicians to maximize efficacy of sunitinib and minimize adverse events. Here, we focus on new insights into molecular mechanisms that may mediate sunitinib-associated adverse events.
\end{abstract}




\section{Introduction}

Tyrosine kinase inhibitors (TKI) are a novel type of drug, designed to target signaling pathways deregulated in cancer by blocking the ATP-binding pocket of specific receptor tyrosine kinases (RTK). TKIs compete with ATP for binding to the intracellular domain of wild-type and/or mutant forms of RTKs and, consequently, inhibit signal transduction.

Although TKIs are designed to target specific RTKs, it is increasingly apparent that many TKIs may display unexpected effects on other kinases and may trigger the appearance of undesirable side effects. The observed incidence of side effects associated with TKIs varies between clinical studies, depending on treatment schedule, dosage, patient characteristics, predisposing factors, secondary diseases, and concurrent administration of other drugs. Moreover, these agents, which do not specifically target tumor cells, are able to act on normal cells, eliciting diverse side effects.

Sunitinib (sunitinib malate; Sutent; Pfizer Inc.) is a targeted TKI able to inhibit members of the RTK families containing a split-kinase domain (Fig. 1; ref. 1). These families include VEGF receptor (VEGFR) types 1 (FLT1), 2 (KDR), and 3 (FLT4); platelet-derived growth factor receptors A and B (PDGFRA and PDGFRB); the stem cell factor receptor (cKIT); FMS-like tyrosine kinase 3 (FLT3); colony-stimulating factor 1 receptor (CSF-1R); and glial cell line-derived neurotrophic factor receptor [RE arranged during transfection (RET); refs. 1-3]. The inhibition of these RTKs blocks signal transduction, thereby affecting various cellular processes, such as tumor growth, tumor progression, angiogenesis, and metastasis (Fig. 1; ref. 4).

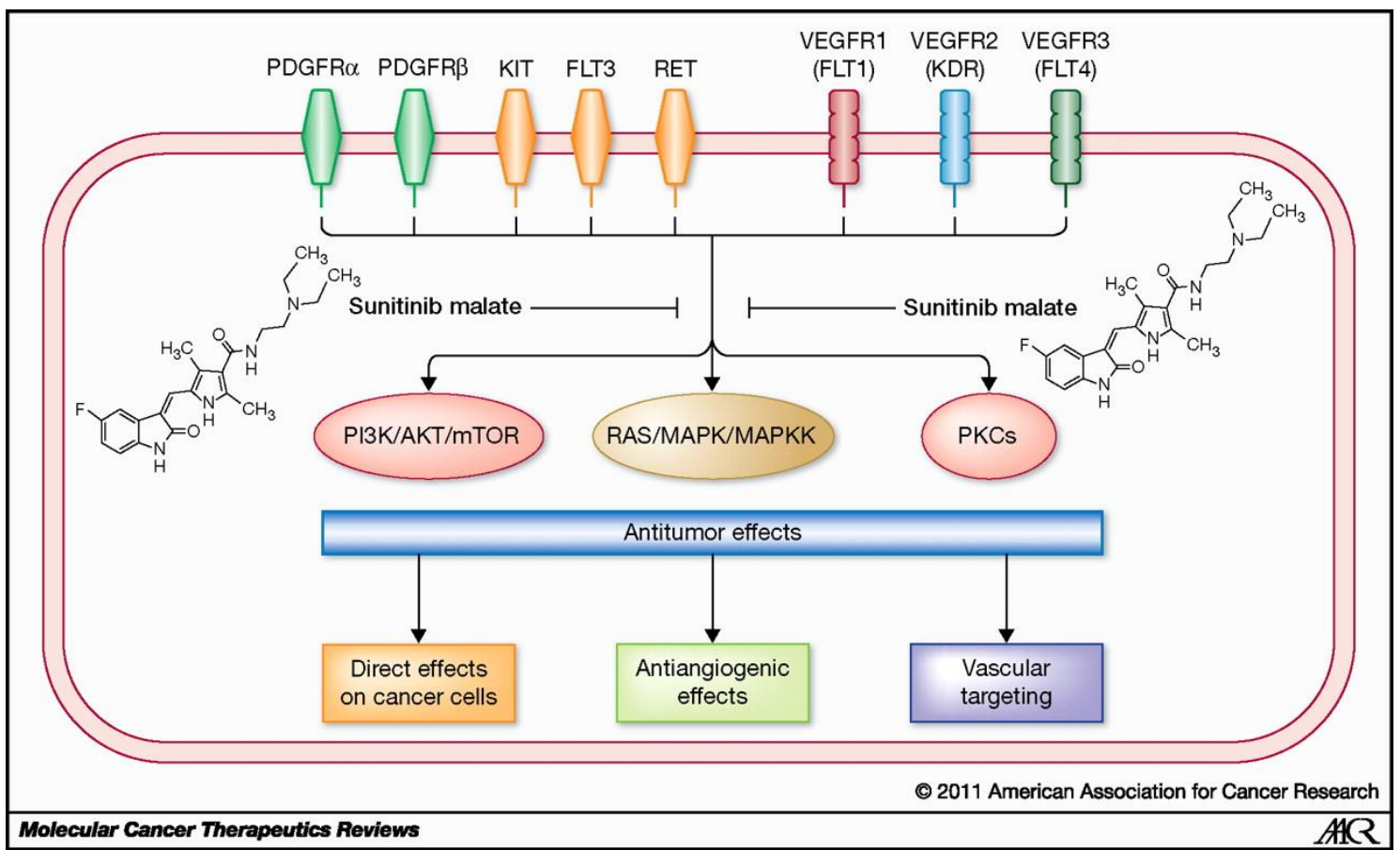

Figure 1. Specific RTKs are blocked by sunitinib (chemical structure shown). Sunitinib is able to block different signaling pathways owing to its action on different RTKs such as PDGFR $\alpha$ and $\beta$, KIT, FLT3, RET, and VEGFRs 1, 2, and 3. Sunitinib inhibition of signaling pathways [phosphoinositide 3-kinase (PI3K)/AKT/mTOR, mitogen-activated protein kinase (MAPK), and $\mathrm{PKC}]$ triggers different antitumor effects (4). 
In a phase III study in treatment-naïve patients with metastatic renal cell carcinoma (RCC), sunitinib significantly doubled median progression-free survival compared with IFN- $\alpha$ and extended median overall survival beyond 2 years $(5,6)$. Sunitinib is approved for the first-line treatment of advanced or metastatic RCC and imatinib-resistant or imatinib-intolerant advanced gastrointestinal stromal tumors (GIST).

Although sunitinib therapy is associated with several well-characterized side effects, the molecular mechanisms underlying these toxicities remain unclear, but they may be related to the additional effect of sunitinib on nonmalignant (normal) cells that also express sunitinib targets, or to the cross-talk between different intracellular signaling pathways. In this review, we examine the current knowledge about the molecular basis of sunitinib side effects, including asthenia, fatigue, hair depigmentation, cardiotoxicity, hypothyroidism, hypertension, dermatologic adverse events, and hematologic and gastrointestinal toxicities.

\section{Molecular Basis of Fatigue and Asthenia}

Fatigue and asthenia are among the most common symptoms experienced by cancer patients, comprising pathologic tiredness, muscle weakness, poor endurance, and impaired motor and cognitive function. Fatigue is one of the most commonly reported side effects of sunitinib treatment, although the degree of fatigue and its impact on quality of life is variable (7). In sunitinib phase III clinical trials, 34\% to $62 \%$ of patients exhibited fatigue of any grade, with $5 \%$ to $15 \%$ reporting grade 3 to 4 fatigue. Asthenia was less common, with an all-grade frequency of $12 \%$ to $25 \%$ and grade 3 to 4 frequency of $3 \%$ to $11 \%$ $(5,6,8,9)$.

The precise clinical mechanisms responsible for causing fatigue and asthenia are unknown but are likely to work through similar pathways. Cancer-related fatigue is known to involve both peripheral (originating in the muscles and related tissues) and central (developing in the CNS) mechanisms, and several possible pathophysiologic pathways have been explored (10). Looking at RCC specifically, a study in diabetic patients showed that sunitinib triggers a decrease in blood glucose levels (11), which may be involved in the induction of fatigue and/or asthenia. However, as both diabetic and nondiabetic patients treated with sunitinib experience fatigue and asthenia (11), other mechanisms may also be involved, as discussed below.

\section{AMP-activated protein kinase}

AMP-activated protein kinase (AMPK) is critical in ensuring a balance between anabolic and catabolic processes within the cell. As such, AMPK functions as an energy sensor protein that is activated in response to an increased cellular AMP-to-ATP ratio, occurring, for example, during nutrient starvation (pooled data in sunitinib-treated RCC show that grade 3-4 anorexia occurs in approximately 3\% of patients; ref. 9) or vigorous muscular exercise. The serine-threonine kinase LKB1 is an important component of the AMPK pathway and modulates AMPK activity (12). Both the AMPK and LKB1 kinases are potential targets for sunitinib (13), which may, therefore, impair the signaling pathway responsible for maintaining the correct energy cellular balance, resulting in fatigue and/or asthenia.

\section{Glucose transporters}

Glucose uptake occurs through facilitative diffusion in a process mediated by plasma membrane glucose transporters. Contractile activity in the muscles triggers glucose uptake in an insulin-independent manner. Nitric oxide synthase (NOS) is also stimulated in muscles in response to contractile activity, increasing nitric oxide (NO) production. Some research suggests that inhibition of NO production can block the ability of exercise to stimulate glucose transport (14). Other studies have shown that endothelial NOS is stimulated in a VEGFR-dependent manner (15). 
In light of these data, sunitinib-mediated inhibition of VEGFR may trigger inhibition of NOS and reduced production of NO, resulting in a reduction in glucose uptake. In addition, as VEGFR can convey its signal through protein kinase C (PKC), sunitinib may indirectly inhibit PKC activity. PKC mediates calcium-mediated glucose uptake in the muscle, and its inhibition could impair insulin-dependent glucose uptake to the muscle.

\section{Circadian rhythm disruption}

Ligands of growth factors may also play a role in circadian rhythm disruption, which can lead to symptom clusters, including fatigue, appetite loss, and sleep disruption (16). Animal studies have identified several ligands of interest, including TGF- $\alpha$, prokinectin-2 (PK2; a molecule closely related to endocrine tissue VEGF), and cardiotropin-like cytokine (Clc; a molecule related to the proinflammatory cytokine interleukin 6). Although currently no clinical data support these associations in patients with RCC, clinical observations have linked elevated levels of TGF- $\alpha$ with fatigue, appetite loss, and flattened circadian rhythms in patients with metastatic colorectal cancer (17).

\section{Molecular Basis of Hair Depigmentation}

Hair depigmentation (gray coloration of scalp or facial hair) has been observed in approximately $10 \%$ of patients treated with sunitinib (18). Hair pigmentation is a result of melanin pigment produced by melanocytes being incorporated into keratinocytes of growing hair. Although the mechanisms causing depigmentation in response to sunitinib treatment are not fully understood, sunitinib does not affect the population of melanocytes associated with hair follicles (19), suggesting that the inhibitory effect of sunitinib takes place at the level of melanocyte function, rather than affecting development or survival.

\section{Signaling pathways during hair pigmentation}

Melanin biosynthesis is mediated by the transcriptional regulator microphthalmia-associated transcription factor (MITF), critical for melanocyte development and differentiation. MITF regulates the transcription of 3 major enzymes involved in melanin biosynthesis, tyrosinase (TYR), tyrosinase-related protein 1 (TYRP1), and tyrosinase-related protein 2 (DCT), as well as its action on other pigmentation factors. MITF is activated by phosphorylation, which is controlled principally by the SCF/KIT signaling pathway (20). Through the inhibition of KIT, sunitinib may prevent activation of MITF, resulting in a lack of enzymes responsible for melanin biosynthesis and, ultimately, impairing hair depigmentation. It is worth noting that hair depigmentation has not been reported with other TKIs, suggesting that kinases other than KIT may also be involved in the process. Alternatively, differences in the occurrence of depigmentation may reflect variations in tissue distribution patterns between the TKIs.

\section{Molecular Basis of Sunitinib Cardiotoxicity}

The reported incidence of cardiotoxicity (primarily left ventricular dysfunction) with sunitinib varies from approximately $11 \%$ to $16 \%$ in the phase III studies in patients with GIST or metastatic RCC, respectively $(4,7,8)$. The molecular mechanisms that may explain sunitinib-induced cardiomyocyte cytotoxicity are reviewed below. 


\section{Angiogenesis}

Among the targets of sunitinib, only PDGFRs are expressed by cardiomyocytes. Although the overexpression of PDGF can promote cardiomyocyte survival (21), endogenous expression of PDGF and its receptors does not seem to have the same effect. Therefore, it is likely that an off-target effect of sunitinib is responsible for the observed cardiotoxicity.

Hypoxia is a critical component of myocardial ischemia. Hypoxia-inducible factor 1 (HIF-1) is a major regulator of the hypoxic response and plays an important role following ischemic infarction, in which supranormal amounts of HIF-1 may have a protective function (22). HIF-1 controls several signaling pathways critical for cellular response to hypoxia, leading to transcriptional activation of angiogenesis-related genes such as VEGF, PDGFB, angiopoietin-1 (ANGPT1), and angiopoietin-2 (ANGPT2). Inhibition of VEGFR by sunitinib, with the ensuing antiangiogenic consequences, may therefore abolish the protective function of HIF-1 in myocardial ischemia, resulting in a potentially lifethreatening situation. In addition, TKI-mediated permanent inhibition of VEGF/VEGFR signaling may impair the normal angiogenic response, compromising the cardiomyocytes' response to pressure (23).

Damage to cardiomyocytes following sunitinib-induced toxicity may also be a result of HIF-1mediated local adaptations to low oxygen tension, such as hypertension triggered by an inducible NOSrelated pathway (see below).

\section{Ribosomal protein S6 kinase and AMP-dependent kinase}

In an earlier review of possible molecular mechanisms of cardiotoxicity of TKIs, Force and colleagues (24) proposed that sunitinib cardiotoxicity might result from off-target inhibition of either AMPK or ribosomal protein S6 kinase (RSK1). Both kinases are predicted to be inhibited by sunitinib at clinically relevant concentrations (12). RSK1 promotes survival through the inhibitory phosphorylation of the proapoptotic factor BCL2-associated agonist of cell death (BAD), and AMPK may transduce prosurvival signals in the heart. Preclinical studies have since shown that sunitinib potently inhibited both AMPK and RSK1 at therapeutically relevant concentrations in vitro and caused dose-dependent damage to cultured myocytes (25). Sunitinib also inhibited phosphorylation of 2 isoforms of the AMPK target, acetylcoenzyme A carboxylase, in myocytes. However, sunitinib did not reduce cellular ATP levels in the myocytes, an effect that would be expected by cellular inhibition of AMPK, nor were the myocytes protected from sunitinib treatment by pretreatment with the AMPK-activating antidiabetic drug metformin (25). Although these results strongly suggest that AMPK inactivation is not responsible for sunitinib-induced cardiotoxicity, metformin generally activates AMPK indirectly. As such, involvement of AMPK, as well as effects on other kinases, cannot be ruled out.

\section{Impaired mitochondrial function}

Another theory to explain sunitinib cardiotoxicity is based on the observation that cardiac tissue uses high amounts of energy and is likely to be very sensitive to mitochondrial damage. Preclinical experiments have shown that sunitinib treatment of cultured cardiomyocytes leads to cytochrome c release into the cytosol (26), which can activate the mitochondrial pathway for cell apoptosis. Consistent with this finding, sunitinib treatment also activated caspase-9, which is an initiator caspase of the mitochondrial apoptotic pathway, and induced apoptosis of cardiomyocytes in vitro (26). Finally, mitochondrial damage was observed in human biopsies and in mice treated with sunitinib (26). However, studies in vitro using isolated rat heart mitochondria failed to find a direct effect of sunitinib on mitochondrial function at clinically relevant concentrations (27). These data do not preclude a secondary effect of sunitinib on mitochondria, which are sensitive to host stress and are often disrupted during necrosis or apoptosis, regardless of cause. Further studies are needed to elucidate the primary cause of sunitinib cardiotoxicity. 


\section{Molecular Basis of Hypothyroidism}

Estimates of the incidence of hypothyroidism in patients treated with sunitinib vary widely. In phase III studies, reported rates have ranged from $4 \%$ to $16 \%(4,7)$, and in a rare prospective observational study, $27 \%$ of patients treated with sunitinib developed sub- or clinical hypothyroidism requiring hormone replacement (28). The mechanisms by which sunitinib affects thyroid function remain unclear but may include antiangiogenic effects $(29,30)$, iodine uptake inhibition (31), destructive thyroiditis (32), inhibition of thyroid peroxidase activity (33), and reduction of vascularity (34) by capillary regression and/or constriction.

The antiangiogenic effect of sunitinib may be responsible for capillary regression $(29,34)$ that enhances toxicity to the thyroid gland. In normal thyroid follicular cells, thyroid-stimulating hormone or thyrotropin (TSH) induces the expression of VEGF and VEGFR, thereby influencing angiogenesis (35). Sunitinib-associated thyroid toxicity may be caused by destruction of the normal thyroid follicular cells due to impaired blood flow and/or inhibition of VEGF signaling.

The observation that iodine uptake is impaired in patients treated with sunitinib (31) prompted the theory that this effect mediates sunitinib-related hypothyroidism. However, preclinical studies found no effect of sunitinib on response of cultured thyroid cells to TSH and a dose-related increase in iodine uptake (36).

Sunitinib is a highly effective inhibitor of the RET/PTC kinase, which plays a role in proliferative signaling pathways (37). Mutations in RET/PTC are thought to be key to the pathogenesis of a significant subset of papillary thyroid carcinomas (38), supporting the idea that the RET pathway plays a role in the normal physiology of the thyroid gland. Therefore, sunitinib-mediated RET/PTC inhibition may impair thyroid activity, inducing hypothyroidism.

Although hypothyroidism is a well-known adverse effect of sunitinib, these abnormal findings in the thyroid gland may serve as potential biomarkers for tumor response to sunitinib (39) and may be related to progression-free survival of patients with metastatic RCC (40).

\section{Molecular Basis of Hypertension}

A considerable body of evidence suggests a link between hypertension and impaired angiogenesis. Hypertension, commonly associated with VEGF inhibitors, may be a biomarker of antitumor efficacy. Recently, hypertension associated with sunitinib treatment was shown to have a significant and independent association with improved clinical outcomes in metastatic RCC (41). Similar results have also been observed for axitinib (42). In hypertensive patients, features associated with angiogenesis include a reduced number of arterioles and capillaries, alterations in the microvascular network, decreased vascular wall flexibility, reduced NOS activity, and increased VEGF in plasma (43). Hypertension may result from depressed angiogenesis at the microcirculation level. A reduction of microvessel density and reduction of vascular areas leads to an increase in peripheral vascular resistance and hypertension.

Sunitinib, as an antiangiogenic drug, may induce hypertension by exerting its influence on mechanisms controlled by nervous and hormonal factors that affect blood vessels. One of the most important factors is $\mathrm{NO}$, an active vasodilator. VEGF enhances transcriptional activity of endothelial NOS (43), suggesting that VEGF could rapidly induce a hypotensive response. Conversely, VEGFsignaling inhibition may partially block NO production in arteriole walls, triggering vascular systemic resistance and hypertension (44). 


\section{Molecular Basis of Dermatologic Toxicity}

Sunitinib frequently causes dermatologic adverse events. In a pooled analysis of published literature (18), sunitinib-induced dermatologic reactions included hand-foot syndrome (19\% of patients), skin rash $(13 \%)$, skin xerosis $(16 \%)$, dermatitis $(8 \%)$, and yellow skin discoloration (28\%; potentially a result of the orange excipient used in sunitinib tablets). Nails with asymptomatic subungual splinter hemorrhages (sometimes accompanied with periungual erythema) have also been reported (45). However, at present, data are lacking from studies undertaken to elucidate the underlying mechanisms causing these toxicities.

\section{Cell signaling and skin toxicity}

The deregulation of signaling pathways, a result of treatment with TKIs, can be used to explain the occurrence of dermatologic side effects and their toxicity. Several experiments have shown the presence of a paracrine feedback loop (46-48), established through VEGF, between keratinocytes and endothelial cells. Furthermore, stromal secretion of PDGF from cell-matrix fibroblasts may also be important in the biology of skin dermis (49). Therefore, the side effects of sunitinib on the skin may be inherent to its action on VEGF and PDGF signaling and to the inhibition of the epidermal growth factor receptor (EGFR) pathway through RAF kinase and/or VEGFR2 inhibition.

Alternatively, VEGFR may be constitutively involved in the formation and repair of delicate spiral capillaries in the skin. The blockade of this receptor through sunitinib might override the physiologic restoration of these capillaries, triggering several side effects. For example, the failure to recover from damage in the capillaries of skin underneath nails may result in subungual splinter hemorrhages (45). In addition, skin toxicity of sunitinib may be a result of its action on the focal adhesion kinase (FAK)/paxilin pathway. As such, sunitinib may trigger changes in cell proliferation and focal adhesion turnover (50).

\section{Molecular Basis of Gastrointestinal Toxicity}

Diarrhea occurs frequently in patients treated with sunitinib, with an incidence in phase III studies ranging between $29 \%$ and 53\% $(4,7)$. Other, less frequent, gastrointestinal adverse events include nausea, stomatitis, vomiting, mucosal inflammation, constipation, and dyspepsia, as well as flatulence and dysgeusia $(4,7,8)$, often mild to moderate in severity.

Very little is known about the mechanisms underlying sunitinib-related gastrointestinal toxicity. Other VEGFR inhibitors commonly lead to diarrhea, nausea, and vomiting, suggesting a class effect (51). However, it seems likely that the mechanisms underlying gastrointestinal toxicity with VEGFR inhibitors, such as sunitinib, seem distinct from those leading to gastrointestinal perforation associated with bevacizumab treatment $(51)$.

\section{Molecular Mechanisms of Hematologic Toxicity}

Most patients with metastatic RCC receiving sunitinib treatment experience some form of hematologic toxicity $(4,5,7)$. This toxicity may be induced by inhibition of the KIT receptor, expressed by hematopoietic progenitor cells (52). In biochemical and in vitro cell-based assays, sunitinib inhibited phosphorylation of the KIT receptor and cellular proliferation (2). In addition, sunitinib inhibits the autophosphorylation of PDGFR and FLT3 (the latter being also expressed primarily on hematopoietic stem cells; refs. 1, 2) and blocks signaling through their downstream effector, extracellular signalregulated kinase 1/2 (ERK1/2; ref. 2). Sunitinib-induced proliferative inhibition by blocking activated tyrosine kinases could result in myelosuppression. In fact, sunitinib treatment results in thrombocytopenia, probably because of myelosuppression. Thrombocytopenia may also be caused by hypertension (53) and by immune thrombocytopenic purpura induced by sunitinib treatment (54). 
However, it is unclear why some patients develop erythrocytosis, whereas others develop anemia. Other erythropoietic pathways may also be involved in the development of hematologic toxicities.

\section{Molecular Mechanisms of Rare Toxicities}

Sunitinib induces several rare toxicities, such as gastrointestinal perforation, colonic pneumatosis, rheumatoid arthritis, encephalopathy syndrome, chronic wounds, and hypophosphatemia.

Perforation of the gastrointestinal wall and/or bleeding of the gastrointestinal mucosa are unique side effects of anti-VEGF therapies, but they are very rare events with VEGFR-directed TKIs (51). As sunitinib blocks VEGFR signaling, it may be responsible for colonic pneumatosis caused by deficiencies in the maintenance of the vessel network.

Although the molecular causes of rheumatoid arthritis are unknown, autoimmunity may play a central role in its progression. The impact of VEGF inhibitors on the immune system has been shown by animal model studies in which VEGF inhibited the development of dendritic cells and increased B lymphocytes and immature myeloid cells (55). However, the effect of sunitinib on the immune system is controversial. A recent article showed that sunitinib impaired proliferation and function of human peripheral $\mathrm{T}$ cells and prevented T-cell-mediated immune response in mice (56), but an earlier publication suggested a lack of effect of sunitinib on primary immune responses (57).

Posterior reversible encephalopathy syndrome (PRES) is characterized by clinical symptoms such as visual loss, including cortical blindness, headache, decreased alertness, and altered mental function. PRES probably results from disruption of cerebral vascular endothelial cells and damage of cerebrovascular autoregulation, leading to edema. Sunitinib may damage the capillary endothelium by targeting VEGFR and PDGFR. VEGF expression in the microvasculature adjacent to choroid plexus epithelium is required for the maintenance of the choroid plexus structure (58); inhibiting VEGF signaling may, therefore, result in loss of the choroid plexus structure. Moreover, in RCC patients with brain metastases, TKIs targeting the VEGFR seem to increase the incidence of fatal intracranial bleeding (59), probably because of destabilization of immature microvasculature.

Patients with chronic wounds (secondary to diabetes, inflammation, or vascular insufficiency) may have altered healing capacity. PDGF and VEGF are essential for wound healing. Degranulation of platelets releases PDGF at the wound site, where its physiologic action is mediated via PDGFRA and PDGFRB. VEGF expression is upregulated in the skin after wounding through its direct action on both endothelial cells and keratinocytes. Treatment with the PDGFR inhibitor imatinib affects wound healing by reducing proliferation and the movement of fibroblasts and pericytes, inhibiting myofibroblast formation and diminishing type I collagen biosynthesis (60); we hypothesize that sunitinib may have a similar effect.

Sunitinib induces an increase in the production of amylase and/or lipase by the exocrine pancreas (4, 8), probably in response to necrosis or capillary regression, which in turn may be caused by the inhibition of VEGFR, Tie, and Eph receptors. Lower levels of blood glucose in diabetic patients treated with sunitinib (9) may be linked to a capillary regression in pancreatic islets and to IGF-I modulation by HIF- $\alpha$ or NF-kB activation, which induces decreased glucose uptake.

Sunitinib-mediated blocking of ATP in active sites of RTKs may trigger the hypophosphatemia observed in patients treated with sunitinib. This blockade forces the cell to incorporate phosphate from other sources to restore the ATP levels. Serum phosphate alterations may also explain hypercalcemia, in which high calcium levels are coupled with low phosphate levels. 


\section{Conclusions}

Targeted therapies represent a new pharmacologic strategy to fight against cancer. New drugs, such as TKIs, targeting specific molecules within important signaling pathways deregulated in cancer have shown notable success.

Multitargeted agents commonly compete with ATP for binding to the ATP pocket of tyrosine kinase receptors, providing potential for the treatment of a broad range of tumors. However, the off-target and broad-spectrum activity associated with these multitargeted drugs confers them with a unique toxicity profile (61-63). At present, 6 targeted agents are approved for the treatment of metastatic RCC in Europe and/or the United States. Examination of the adverse-event profile associated with each of these agents shows some important similarities as well as differences (Table 1; refs. 6, 64-68). These differences may be partly attributable to the differences in RTK potencies when binding to various targets (Table 2; refs. 69-71).

Table 1. Selected all-grade adverse events associated with the targeted agents sunitinib, sorafenib, bevacizumab/IFN- $\alpha$, temsirolimus, everolimus, and pazopanib, approved in Europe and/or the United States for the treatment of metastatic RCC

\begin{tabular}{|c|c|c|c|c|c|c|}
\hline Adverse event (\%) & Sunitinib & Sorafenib & $\begin{array}{l}\text { Bevacizumab plus IFN- } \\
\alpha\end{array}$ & Temsirolimus & Everolimus & Pazopanib \\
\hline Anorexia & 34 & 16 & 36 & 32 & 16 & 22 \\
\hline Asthenia & 20 & - & 32 & 51 & 18 & 14 \\
\hline Diarrhea & 61 & 43 & 20 & 27 & 17 & 52 \\
\hline Dyspnea & 10 & 14 & 13 & 28 & 8 & - \\
\hline Fatigue & 54 & 37 & 33 & - & 20 & 19 \\
\hline Hand-foot syndrome & 29 & 30 & - & - & - & - \\
\hline Hyperglycemia & - & - & - & 26 & 50 & 41 \\
\hline Proteinuria & - & - & 18 & - & - & - \\
\hline Hypertension & 20 & 17 & 26 & - & - & 40 \\
\hline Anemia & 79 & 8 & 10 & 45 & 91 & - \\
\hline Lymphopenia & 68 & - & - & - & 42 & 31 \\
\hline Neutropenia & 77 & - & 7 & 7 & 11 & 34 \\
\hline Thrombocytopenia & 68 & - & 6 & 14 & 20 & 32 \\
\hline Leucopenia & 78 & - & - & 6 & 26 & 32 \\
\hline $\begin{array}{l}\text { Discontinuations due to AEs } \\
(\%)\end{array}$ & 19 & 10 & 28 & 7 & 10 & NR \\
\hline
\end{tabular}

NOTE: Data from refs. 6, 64-68.

Abbreviations: AE, adverse event; NR, not reported. 
Table 2. Potency of selected agents for various tyrosine kinase receptors

\begin{tabular}{|c|c|c|c|c|}
\hline \multicolumn{5}{|c|}{$\mathrm{IC}_{50}(\mathrm{nM})^{\mathrm{a}}$} \\
\hline Target & Sunitinib & Sorafenib & Pazopanib & Axitinib \\
\hline VEGFR-1 & 2 & - & 10 & 1.2 \\
\hline VEGFR-2 & 10 & 90 & 30 & 0.25 \\
\hline VEGFR-3 & 17 & 20 & 47 & 0.29 \\
\hline PDGFR- $\beta$ & 8 & 57 & 84 & 1.7 \\
\hline EGFR & 880 & 58 & - & - \\
\hline c-KIT & 10 & 68 & 74 & 1.6 \\
\hline FGF-1R & 880 & 580 & 14 & 230 \\
\hline FLT-3 & 14 & 58 & - & - \\
\hline Raf-1 & - & 6 & - & - \\
\hline CSF-1R & 100 & - & - & - \\
\hline
\end{tabular}

NOTE: Data from refs. 69-71.

Abbreviations: FGF, fibroblast growth factor.

${ }^{\mathrm{a}} \mathrm{IC}_{50}$ represents the concentration of a drug that is required to achieve $50 \%$ inhibition of the enzyme in a biochemical assay.

For some toxicities, differentiating between the target and off-target effects of sunitinib is not straightforward. Comparing the toxicities associated with sunitinib with those associated with the selective anti-VEGF monoclonal antibody bevacizumab may be useful. For example, grade 3 to 4 left ventricular dysfunction has been observed to occur with greater frequency with sunitinib treatment than with either bevacizumab or sorafenib $(0.3 \%, 1.4 \%$, and $0.05 \%$ in phase I-III studies), suggesting a potential off-target effect (72). One potential explanation may be that the reduced left ventricular ejection fraction is a result of sunitinib-induced hypothyroidism (24). Another example is that dermatologic toxicities, specifically hand-foot skin reactions, occur much less commonly in patients treated with bevacizumab than in those treated with sunitinib. However, rather than hinting toward a potential offtarget effect of sunitinib, evidence suggests that dermatologic toxicities in patients treated with bevacizumab may be modulated by the presence of certain VEGF2 alleles (73).

Currently no data from randomized clinical trials directly compare the activity and toxicity profile of various multitargeted agents. Two phase III studies comparing sunitinib with pazopanib (COMPARZ:NCT00720941) and axitinib with sorafenib, respectively, are ongoing; early results are expected by 2011 (74). Increased understanding of the pathogenesis of sunitinib-associated side effects may allow rational administration to patients. Optimizing sunitinib treatment is essential, as increased exposure is associated with improved clinical benefit (75). In a meta-analysis of data from patients with metastatic RCC and GIST, increased exposure was associated with longer time to progression, overall survival, higher probability of a response, and greater decrease in tumor size, but also with more adverse events, albeit of mild-to-moderate intensity in most patients (75).

To date, only retrospective analyses about the association between occurrence of a defined adverse event and clinical outcome are available. In both axitinib and sunitinib studies, a relationship has been shown between incidence of hypertension and clinical outcome endpoints, including overall response rate and progression-free and overall survival $(41,42)$.

Additionally, several retrospective studies have shown a relationship between the presence of hypothyroidism and improved antitumor efficacy in patients treated with sunitinib $(34,39,40)$. Although further studies are required, the abnormalities in thyroid function or blood pressure following treatment with sunitinib may be potential biomarkers for tumor response to multitargeted agents. 
Understanding the molecular basis of sunitinib toxicity will help explain the mechanisms responsible for inducing undesirable side effects with the broader class of targeted agents, ultimately allowing the design of more effective targeted anticancer therapies with an improved safety profile.

\section{Disclosure of Potential Conflicts of Interest}

No potential conflicts of interest were disclosed.

\section{Grant Support}

M. Blanco-Calvo is supported by a grant from Instituto de Salud Carlos III; A. Figueroa is supported by the Isidro Parga Pondal Program, Xunta de Galicia, Spain. Medical writing support was provided by Minal Kotecha at ACUMED (Tytherington, UK) and was funded by Pfizer, Inc.

\section{References}

1. Mendel DB, Laird AD, Xin X, Louie SG, Christensen JG, Li G, et al.In vivo antitumor activity of SU11248, a novel tyrosine kinase inhibitor targeting vascular endothelial growth factor and platelet-derived growth factor receptors: determination of a pharmacokinetic/pharmacodynamic relationship. Clin Cancer Res 2003;9:327-37.

2. Abrams TJ, Lee LB, Murray LJ, Pryer NK, Cherrington JM. SU11248 inhibits KIT and platelet-derived growth factor receptor beta in preclinical models of human small cell lung cancer. Mol Cancer Ther 2003;2:471-8.

3. O'Farrell AM, Abrams TJ, Yuen HA, Ngai TJ, Louie SG, Yee KW, et al.SU11248 is a novel FLT3 tyrosine kinase inhibitor with potent activity in vitro and in vivo. Blood 2003;101:3597-605.

4. Faivre S, Demetri G, Sargent W, Raymond E. Molecular basis for sunitinib efficacy and future clinical development. Nat Rev Drug Discov 2007;6:734-45.

5. Motzer RJ, Hutson TE, Tomczak P, Michaelson MD, Bukowski RM, Rixe O, et al.Sunitinib versus interferon alfa in metastatic renal-cell carcinoma. N Engl J Med 2007;356:115-24.

6. Motzer RJ, Hutson TE, Tomczak P, Michaelson MD, Bukowski RM, Oudard S, et al.Overall survival and updated results for sunitinib compared with interferon alfa in patients with metastatic renal cell carcinoma. J Clin Oncol 2009;27:3584-90.

7. Cella D, Michaelson MD, Bushmakin AG, Cappelleri JC, Charbonneau C, Kim ST, et al.Health-related quality of life in patients with metastatic renal cell carcinoma treated with sunitinib vs interferon-alpha in a phase III trial: final results and geographical analysis. Br J Cancer 2010;102:658-64.

8. Demetri GD, van Oosterom AT, Garrett CR, Blackstein ME, Shah MH, Verweij J, et al.Efficacy and safety of sunitinib in patients with advanced gastrointestinal stromal tumour after failure of imatinib: a randomised controlled trial. Lancet 2006;368:1329-38.

9. eMC. Leatherhead (Surrey, England): Datapharm. Pfizer Limited; SUTENT, Summary of product characteristics; December 2010. Available from: http://www.medicines.org.uk/EMC/medicine/18531/SPC/SUTENT+12.5mg\%2c+25mg\%2c+ $37.5 \mathrm{mg}+$ and $+50 \mathrm{mg}+$ Hard+Capsules/.

10. Barsevick A, Frost M, Zwinderman A, Hall P, Halyard M. GENEQOL Consortium. I'm so tired: biological and genetic mechanisms of cancer-related fatigue. Qual Life Res 2010;19:1419-27.

11. Billemont B, Medioni J, Taillade L, Helley D, Meric JB, Rixe O, et al.Blood glucose levels in patients with metastatic renal cell carcinoma treated with sunitinib. Br J Cancer 2008;99:1380-2.

12. Carretero J, Medina PP, Blanco R, Smit L, Tang M, Roncador G, et al.Dysfunctional AMPK activity, signalling through mTOR and survival in response to energetic stress in LKB1deficient lung cancer. Oncogene 2007;26:1616-25. 
13. Fabian MA, Biggs WH 3rd., Treiber DK, Atteridge CE, Azimioara MD, Benedetti MG, et al.A small molecule-kinase interaction map for clinical kinase inhibitors. Nat Biotechnol 2005;23:329-36.

14. Richter EA, Derave W, Wojtaszewski JF. Glucose, exercise and insulin: emerging concepts. J Physiol 2001;535:313-22.

15. Lahdenranta J, Hagendoorn J, Padera TP, Hoshida T, Nelson G, Kashiwagi S, et al.Endothelial nitric oxide synthase mediates lymphangiogenesis and lymphatic metastasis. Cancer Res 2009;69:2801-8.

16. Rich TA. Symptom clusters in cancer patients and their relation to EGFR ligand modulation of the circadian axis. J Support Oncol 2007;5:167-74, discussion 176-7.

17. Rich T, Innominato PF, Boerner J, Mormont MC, Iacobelli S, Baron B, et al.Elevated serum cytokines correlated with altered behavior, serum cortisol rhythm, and dampened 24-hour rest-activity patterns in patients with metastatic colorectal cancer. Clin Cancer Res 2005;11:1757-64.

18. Rosenbaum SE, Wu S, Newman MA, West DP, Kuzel T, Lacouture ME. Dermatological reactions to the multitargeted tyrosine kinase inhibitor sunitinib. Support Care Cancer 2008;16:557-66.

19. Moss KG, Toner GC, Cherrington JM, Mendel DB, Laird AD. Hair depigmentation is a biological readout for pharmacological inhibition of KIT in mice and humans. J Pharmacol Exp Ther 2003;307:476-80.

20. Giebel LB, Spritz RA. Mutation of the KIT (mast/stem cell growth factor receptor) protooncogene in human piebaldism. Proc Natl Acad Sci U S A 1991;88:8696-9.

21. Hsieh PC, MacGillivray C, Gannon J, Cruz FU, Lee RT. Local controlled intramyocardial delivery of platelet-derived growth factor improves postinfarction ventricular function without pulmonary toxicity. Circulation 2006;114:637-44.

22. Kido M, Du L, Sullivan CC, Li X, Deutsch R, Jamieson SW, et al.Hypoxia-inducible factor 1alpha reduces infarction and attenuates progression of cardiac dysfunction after myocardial infarction in the mouse. J Am Coll Cardiol 2005;46:2116-24.

23. Izumiya Y, Shiojima I, Sato K, Sawyer DB, Colucci WS, Walsh K. Vascular endothelial growth factor blockade promotes the transition from compensatory cardiac hypertrophy to failure in response to pressure overload. Hypertension 2006;47:887-93.

24. Force T, Krause DS, Van Etten RA. Molecular mechanisms of cardiotoxicity of tyrosine kinase inhibition. Nat Rev Cancer 2007;7:332-44.

25. Hasinoff BB, Patel D, O'Hara KA. Mechanisms of myocyte cytotoxicity induced by the multiple receptor tyrosine kinase inhibitor sunitinib. Mol Pharmacol 2008;74:1722-8.

26. Chu TF, Rupnick MA, Kerkela R, Dallabrida SM, Zurakowski D, Nguyen L, et al.Cardiotoxicity associated with tyrosine kinase inhibitor sunitinib. Lancet 2007;370:2011-9.

27. Will Y, Dykens JA, Nadanaciva S, Hirakawa B, Jamieson J, Marroquin LD, et al.Effect of the multitargeted tyrosine kinase inhibitors imatinib, dasatinib, sunitinib, and sorafenib on mitochondrial function in isolated rat heart mitochondria and $\mathrm{H} 9 \mathrm{c} 2$ cells. Toxicol Sci 2008;106:153-61.

28. Wolter P, Stefan C, Decallonne B, Dumez H, Bex M, Carmeliet P, et al.The clinical implications of sunitinib-induced hypothyroidism: a prospective evaluation. Br J Cancer 2008;99:448-54.

29. Jebreel A, England J, Bedford K, Murphy J, Karsai L, Atkin S. Vascular endothelial growth factor (VEGF), VEGF receptors expression and microvascular density in benign and malignant thyroid diseases. Int J Exp Pathol 2007;88:271-7.

30. Yamada E, Yamazaki K, Takano K, Obara T, Sato K. Iodide inhibits vascular endothelial growth factor-A expression in cultured human thyroid follicles: a microarray search for effects of thyrotropin and iodide on angiogenesis factors. Thyroid 2006;16:545-54.

31. Mannavola D, Coco P, Vannucchi G, Bertuelli R, Carletto M, Casali PG, et al.A novel tyrosine-kinase selective inhibitor, sunitinib, induces transient hypothyroidism by blocking iodine uptake. J Clin Endocrinol Metab 2007;92:3531-4.

32. Desai J, Yassa L, Marqusee E, George S, Frates MC, Chen MH, et al.Hypothyroidism after sunitinib treatment for patients with gastrointestinal stromal tumors. Ann Intern Med 2006;145:660-4.

33. Wong E, Rosen LS, Mulay M, Vanvugt A, Dinolfo M, Tomoda C, et al.Sunitinib induces hypothyroidism in advanced cancer patients and may inhibit thyroid peroxidase activity. Thyroid 2007; 17:351-5.

34. Makita N, Miyakawa M, Fujita T, Iiri T. Sunitinib induces hypothyroidism with a markedly reduced vascularity. Thyroid 2010;20:323-6. 
35. Hoffmann S, Hofbauer LC, Scharrenbach V, Wunderlich A, Hassan I, Lingelbach S, et al.Thyrotropin (TSH)-induced production of vascular endothelial growth factor in thyroid cancer cells in vitro: evaluation of TSH signal transduction and of angiogenesis-stimulating growth factors. J Clin Endocrinol Metab 2004;89:6139-45.

36. Salem AK, Fenton MS, Marion KM, Hershman JM. Effect of sunitinib on growth and function of FRTL-5 thyroid cells. Thyroid 2008;18:631-5.

37. Kim DW, Jo YS, Jung HS, Chung HK, Song JH, Park KC, et al.An orally administered multitarget tyrosine kinase inhibitor, SU11248, is a novel potent inhibitor of thyroid oncogenic RET/papillary thyroid cancer kinases. J Clin Endocrinol Metab 2006;91:4070-6

38. Jhiang SM. The RET proto-oncogene in human cancers. Oncogene 2000;19:5590-7.

39. Shinohara N, Takahashi T, Kamishima H, Ikushima H, Sazawa A, Kanayama H. Efficacy and thyroidal effects of sunitinib in Japanese patients with metastatic renal cell carcinoma: Hypothyroidism and thyroid atrophy as potential biomarkers for sunitinib? J Clin Oncol 2009;27:e16097.

40. Sabatier R, Gravis G, Deville J, Salem N, Brunelle S, Walz J. Hypothyroidism and survival during sunitinib therapy in metastatic renal cell cancer: A prospective observational analysis. American Society for Clinical Oncology 2009 Genitourinary Cancers Symposium 2009; Abstract 317.

41. Rini BI, Cohen DP, Lu D, Chen I, Hariharan S, Gore ME, et al.Hypertension (HTN) as a biomarker of efficacy in patients (pts) with metastatic renal cell carcinoma (mRCC) treated with sunitinib. In: Proceedings of the American Society for Clinical Oncology 2010 Genitourinary Cancers Symposium 2010 Mar 5-7; San Francisco. Alexandria (VA): ASCO; 2010. Abstract nr 312.

42. Rixe O, Dutcher J, Motzer R, Wilding G, Stadler WM, Garrett M, et al.Diastolic blood pressure (dBP) and pharmacokinetics $(\mathrm{PK})$ as predictors of axitinib efficacy in metastatic renal cell cancer (mRCC). J Clin Oncol 2009;27:5045.

43. Hood JD, Meininger CJ, Ziche M, Granger HJ. VEGF upregulates ecNOS message, protein, and NO production in human endothelial cells. Am J Physiol 1998;274:H1054-8.

44. Sane DC, Anton L, Brosnihan KB. Angiogenic growth factors and hypertension. Angiogenesis 2004;7:193-201.

45. Robert C, Soria JC, Spatz A, Le Cesne A, Malka D, Pautier P, et al.Cutaneous side-effects of kinase inhibitors and blocking antibodies. Lancet Oncol 2005;6:491-500.

46. Detmar M, Yeo KT, Nagy JA, Van de Water L, Brown LF, Berse B, et al.Keratinocytederived vascular permeability factor (vascular endothelial growth factor) is a potent mitogen for dermal microvascular endothelial cells. J Invest Dermatol 1995;105:44-50.

47. Rossiter H, Barresi C, Pammer J, Rendl M, Haigh J, Wagner EF, et al.Loss of vascular endothelial growth factor a activity in murine epidermal keratinocytes delays wound healing and inhibits tumor formation. Cancer Res 2004;64:3508-16.

48. Wilgus TA, Matthies AM, Radek KA, Dovi JV, Burns AL, Shankar R, et al.Novel function for vascular endothelial growth factor receptor-1 on epidermal keratinocytes. Am J Pathol 2005;167:1257-66.

49. Zhang JZ, Maruyama K, Ono I, Kaneko F. Production and secretion of platelet-derived growth factor $\mathrm{AB}$ by cultured human keratinocytes: regulatory effects of phorbol 12-myristate 13-acetate, etretinate, 1,25-dihydroxyvitamin D3, and several cytokines. J Dermatol 1995;22:305-9.

50. de Boüard S, Herlin P, Christensen JG, Lemoisson E, Gauduchon P, Raymond E, et al.Antiangiogenic and anti-invasive effects of sunitinib on experimental human glioblastoma. Neuro-oncol 2007;9:412-23.

51. Roodhart JM, Langenberg MH, Witteveen E, Voest EE. The molecular basis of class side effects due to treatment with inhibitors of the VEGF/VEGFR pathway. Curr Clin Pharmacol 2008;3:132-43.

52. Brandt J, Briddell RA, Srour EF, Leemhuis TB, Hoffman R. Role of c-kit ligand in the expansion of human hematopoietic progenitor cells. Blood 1992;79:634-41.

53. Kapiteijn E, Brand A, Kroep J, Gelderblom H. Sunitinib induced hypertension, thrombotic microangiopathy and reversible posterior leukencephalopathy syndrome. Ann Oncol 2007; 18:1745-7.

54. Trinkaus M, Trudeau M, Callum J. Drug-induced immune thrombocytopenic purpura secondary to sunitinib. Curr Oncol 2008;15:152-4.

55. Gabrilovich D, Ishida T, Oyama T, Ran S, Kravtsov V, Nadaf S, et al.Vascular endothelial growth factor inhibits the development of dendritic cells and dramatically affects the differentiation of multiple hematopoietic lineages in vivo. Blood 1998;92:4150-66. 
56. Gu Y, Zhao W, Meng F, Qu B, Zhu X, Sun Y, et al.Sunitinib impairs the proliferation and function of human peripheral $\mathrm{T}$ cell and prevents T-cell-mediated immune response in mice. Clin Immunol 2010;135:55-62.

57. Hipp MM, Hilf N, Walter S, Werth D, Brauer KM, Radsak MP, et al.Sorafenib, but not sunitinib, affects function of dendritic cells and induction of primary immune responses. Blood 2008;111:5610-20.

58. Maharaj AS, Walshe TE, Saint-Geniez M, Venkatesha S, Maldonado AE, Himes NC, et al.VEGF and TGF-beta are required for the maintenance of the choroid plexus and ependyma. J Exp Med 2008;205:491-501.

59. Pouessel D, Culine S. High frequency of intracerebral hemorrhage in metastatic renal carcinoma patients with brain metastases treated with tyrosine kinase inhibitors targeting the vascular endothelial growth factor receptor. Eur Urol 2008;53:376-81.

60. Rajashekar A, Perazella MA, Crowley S. Systemic diseases with renal manifestations. Prim Care 2008;35:297-328, vi-vii.

61. De Luca A, Carotenuto A, Rachiglio A, Gallo M, Maiello MR, Aldinucci D, et al.The role of the EGFR signaling in tumor microenvironment. J Cell Physiol 2008;214:559-67.

62. Steeghs N, Gelderblom H, Roodt JO, Christensen O, Rajagopalan P, Hovens M, et al.Hypertension and rarefaction during treatment with telatinib, a small molecule angiogenesis inhibitor. Clin Cancer Res 2008;14:3470-6.

63. Zhou J, Goh BC, Albert DH, Chen CS. ABT-869, a promising multi-targeted tyrosine kinase inhibitor: from bench to bedside. J Hematol Oncol 2009;2:33.

64. Escudier B, Eisen T, Stadler WM, Szczylik C, Oudard S, Siebels M, et al.TARGET Study Group. Sorafenib in advanced clear-cell renal-cell carcinoma. N Engl J Med 2007;356:12534.

65. Escudier B, Bellmunt J, Négrier S, Bajetta E, Melichar B, Bracarda S, et al.Phase III trial of bevacizumab plus interferon alfa-2a in patients with metastatic renal cell carcinoma (AVOREN): final analysis of overall survival. J Clin Oncol 2010;28:2144-50.

66. Hudes G, Carducci M, Tomczak P, Dutcher J, Figlin R, Kapoor A, et al.Global ARCC Trial. Temsirolimus, interferon alfa, or both for advanced renal-cell carcinoma. N Engl J Med 2007;356:2271-81.

67. Motzer RJ, Escudier B, Oudard S, Hutson TE, Porta C, Bracarda S, et al.RECORD-1 Study Group. Efficacy of everolimus in advanced renal cell carcinoma: a double-blind, randomised, placebo-controlled phase III trial. Lancet 2008;372:449-56.

68. Sternberg CN, Davis ID, Mardiak J, Szczylik C, Lee E, Wagstaff J, et al.Pazopanib in locally advanced or metastatic renal cell carcinoma: results of a randomized phase III trial. J Clin Oncol 2010;28:1061-8.

69. Kumar R, Knick VB, Rudolph SK, Johnson JH, Crosby RM, Crouthamel MC, et al.Pharmacokinetic-pharmacodynamic correlation from mouse to human with pazopanib, a multikinase angiogenesis inhibitor with potent antitumor and antiangiogenic activity. Mol Cancer Ther 2007;6:2012-21.

70. Schmidinger M, Bellmunt J. Plethora of agents, plethora of targets, plethora of side effects in metastatic renal cell carcinoma. Cancer Treat Rev 2010;36:416-24.

71. Wilhelm SM, Carter C, Tang L, Wilkie D, McNabola A, Rong H, et al.BAY 43-9006 exhibits broad spectrum oral antitumor activity and targets the RAF/MEK/ERK pathway and receptor tyrosine kinases involved in tumor progression and angiogenesis. Cancer Res 2004;64:7099109.

72. Vaklavas C, Lenihan D, Kurzrock R, Tsimberidou AM. Anti-vascular endothelial growth factor therapies and cardiovascular toxicity: what are the important clinical markers to target? Oncologist 2010;15:130-41.

73. Jain L, Sissung TM, Danesi R, Kohn EC, Dahut WL, Kummar S, et al.Hypertension and hand-foot skin reactions related to VEGFR2 genotype and improved clinical outcome following bevacizumab and sorafenib. J Exp Clin Cancer Res 2010;29:95.

74. Rini BI, Escudier B, Tomczak P, Kaprin A, Hutson TE, Szczylik C, et al.Axitinib versus sorafenib as second-line therapy for metastatic renal cell carcinoma (mRCC): Results of phase III AXIS trial. J Clin Oncol 2011;29 (suppl; abstr 4503).

75. Houk BE, Bello CL, Poland B, Rosen LS, Demetri GD, Motzer RJ. Relationship between exposure to sunitinib and efficacy and tolerability endpoints in patients with cancer: results of a pharmacokinetic/pharmacodynamic meta-analysis. Cancer Chemother Pharmacol 2010;66:357-71. 